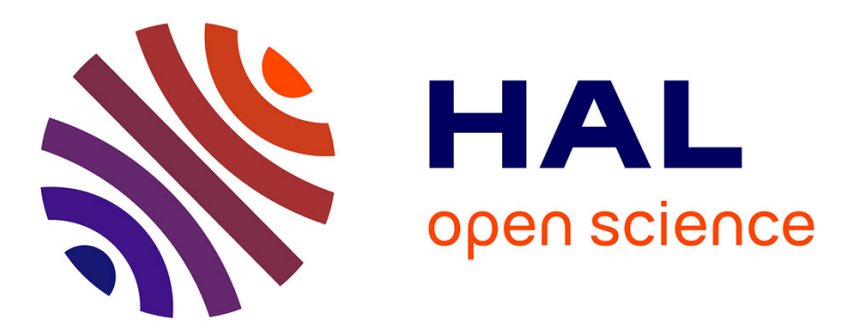

\title{
Dioramas ethnographiques et unités écologiques : La mise en scène de la vie quotidienne au musée d'Ethnographie du Trocadéro et au musée national des Arts et Traditions populaires
}

Jean-Roch Bouiller, Marie-Charlotte Calafat

\section{To cite this version:}

Jean-Roch Bouiller, Marie-Charlotte Calafat. Dioramas ethnographiques et unités écologiques : La mise en scène de la vie quotidienne au musée d'Ethnographie du Trocadéro et au musée national des Arts et Traditions populaires. Culture et Musées, 2018, 32, pp.131-158. 10.4000/culturemusees.2473 . hal-03254369

\section{HAL Id: hal-03254369 \\ https://hal.science/hal-03254369}

Submitted on 8 Jun 2021

HAL is a multi-disciplinary open access archive for the deposit and dissemination of scientific research documents, whether they are published or not. The documents may come from teaching and research institutions in France or abroad, or from public or private research centers.
L'archive ouverte pluridisciplinaire $\mathbf{H A L}$, est destinée au dépôt et à la diffusion de documents scientifiques de niveau recherche, publiés ou non, émanant des établissements d'enseignement et de recherche français ou étrangers, des laboratoires publics ou privés. 


\section{OpenEdition}

Journals

\section{Culture \& Musées}

Muséologie et recherches sur la culture

$32 \mid 2018$

L'art du diorama (1700-2000)

Dossier

\section{Dioramas ethnographiques et} unités écologiques : La mise en scène de la vie quotidienne au musée d'Ethnographie du Trocadéro et au musée national des Arts et Traditions populaires

Ethnographic dioramas and ecological units: Staging daily life at the Trocadero Museum of ethnography and the National Museum of popular arts and traditions

Dioramas etnográficos y unidades ecológicas: La puesta en escena de la vida cotidiana en el Museo Etnográfico del Trocadero y en el Museo Nacional de Artes y Tradiciones Populares

Jean-Roch Bouiller et Marie-Charlotte Calafat

p. $131-158$

https://doi.org/10.4000/culturemusees.2473

\section{Résumés}

Français English Español

Cet article a pour objet l'étude et la mise en perspective historique des dioramas et unités écologiques réalisés dans les deux musées nationaux français consacrés à l'ethnographie de la France, entre 1878 et 1978 : le musée d'Ethnographie du Trocadéro (MET) puis le musée national des Arts et Traditions populaires (MNATP), à Paris. Il essaye tout d'abord de saisir l'esprit du temps qui les a inspirés et dont ils sont les témoins : influence des Expositions universelles, des panoramas, des musées de mannequins de cire, des recherches archéologiques, des recherches historiques conduisant aux period rooms, etc. Il cherche ensuite à comprendre les liens ambigus que ces dispositifs scénographiques entretiennent avec la réalité, sa représentation et l'illusion de sa restitution fidèle. Il souligne enfin les points de convergences de ces éléments identitaires, propres aux dioramas et aux unités écologiques, avec le développement de l'installation dans le champ de l'art contemporain, dans la deuxième moitié du XXe siècle. 
This article studies in historical perspective dioramas and ecological units produced in two French national museums devoted to French ethnography between 1878 and 1978: the Musée d'ethnographie du Trocadéro (MET) and the Musée national des arts et traditions populaires (MNATP) in Paris. First of all, we try to understand which elements of context could inspire them: Universal Exhibitions, panoramas, wax model museums, archaeological research, historical research and period rooms, etc. We also are interested in ambiguous links between these scenographic devices and reality, representation of reality and illusion of its faithful reproduction. Finally, it highlights the points of convergence between dioramas and ecological units with the development of installation in the field of contemporary art in the second half of the 2oth century.

Este artículo tiene como objetivo estudiar y poner en perspectiva histórica los dioramas y unidades ecológicas realizadas en los dos museos nacionales franceses dedicados a la etnografía de Francia, entre 1878 y 1978: el Museo Etnográfico del Trocadero (MET) y el Museo Nacional de Artes y Tradiciones Populares (MNATP) en París. En primer lugar, trata de captar el espíritu de la época que los inspiró y de los cuales son testigos: la influencia de las Exposiciones universales, de los panoramas, de los museos de figuras de cera, de investigaciones arqueológicas e investigaciones históricas que conducen a los period room (salas de época), etc. Luego busca comprender los vínculos ambiguos que estos dispositivos escenográficos mantienen con la realidad, su representación y la ilusión de su reproducción fiel. Finalmente, subraya los puntos de convergencia de estos elementos identitarios, específicos de los dioramas y unidades ecológicas, con el desarrollo de la instalación en el campo del arte contemporáneo, en la segunda mitad del siglo XX.

\section{Entrées d'index}

Mots-clés : dioramas, unités écologiques, ethnographie, muséographie, scénographie

Keywords: dioramas, ecological units, ethnography, museography, scenography

Palabras clave: dioramas, unidades ecológicas, etnografía, museografía, escenografía

\section{Notes de la rédaction}

Manuscrit reçu le 18 janvier 2018

Version révisée reçue le 31 août 2018

Article accepté pour publication le 21 septembre 2018

\section{Texte intégral}

1 Les dioramas sont à la mode. Restaurés par les institutions, réhabilités par les muséologues, étudiés par les chercheurs, parodiés ou sublimés par les artistes contemporains, comme dans la récente exposition Dioramas au Palais de Tokyo (Le Bon et al., 2016), ils semblent sortis de la torpeur où les avait plongés une supposée désuétude. Reflets du monde réel, ils apparaissent aujourd'hui comme le paroxysme de ce qui fait l'essence du musée : un miroir du monde, des sociétés, du rapport de l'homme à son environnement immédiat.

2 À une époque où certains musées ont le vent en poupe tandis que d'autres sont menacés de disparition, où le média exposition connaît des développements et des expérimentations sans précédent, où les études sur les musées n'ont jamais été aussi nombreuses et où le tournant numérique annonce de nouveaux bouleversements, l'histoire des dioramas peut être perçue comme un sismographe des métamorphoses de l'institution muséale. C'est tout particulièrement perceptible si l'on observe leur évolution au sein des musées nationaux français consacrés à l'ethnographie de la France durant le premier siècle de leur histoire, entre 1878 et 1978 : d'abord au musée d'Ethnographie du Trocadéro (MET), à Paris, de 1878 à 1937 ; puis au musée national des Arts et Traditions populaires (MNATP), en chantier entre 1937 et 1972, inauguré en deux temps en 1972 et 1975. Le corpus étudié recouvre donc les dix dioramas du MET et les six unités écologiques de la galerie culturelle du MNATP, à travers les photographies et les éléments matériels conservés ${ }^{1}$. Les limites de l'exercice sont liées au fait qu'on ne peut s'appuyer que sur ces rares traces. Les archives et la littérature spécialisée dans le domaine muséographique (journaux, catalogues, bulletins...) offrent, à travers une lecture historique, des éléments de compréhension des intentions ainsi que des conditions de production et de réception de ces dispositifs scénographiques. 
3 Observer les mutations des différents dioramas réalisés dans ce contexte permet tout d'abord de mieux cerner leur genèse et leurs sources d'inspiration. Cet exercice met également en évidence la cristallisation de trois ingrédients, apparemment contradictoires, dont le musée est pourtant le fruit : le contenu scientifique qui constitue son fonds, l'idéologie politique qui le sous-tend et le dispositif scénographique qui en offre une grille de lecture concrète et spectaculaire. On peut légitimement se demander comment la coexistence de ces trois composantes évolue sur une durée d'un siècle, dans des contextes historiques différents. La subtile négociation entre des notions telles que réalité, vrai, faux, authentique et vraisemblable se trouve au cœur de cette cohabitation. On peut, enfin, soumettre à l'analyse comparative les différentes formes de dioramas auxquelles les deux musées ont donné lieu, en cherchant à faire émerger ce qu'elles ont de fondamentalement semblable et différent. De ces constatations pourront émerger des considérations sur les limites et les atouts de la forme dioramique au sein du musée.

\section{Genèse et sources d'inspiration}

4 Les Expositions universelles ont influencé la muséographie de la fin du XIXe siècle : c'est dans cette continuité que le diorama est arrivé dans les musées ethnographiques français, à partir des premières expérimentations d'Artur Hazelius, du Nordiska Museet, en Suède, présentées et diffusées lors de l'Exposition universelle de 1878 à Paris (DeGroff, 2012 : 113-114 ; Dias, 1991 : 175-203 ; Séréna-Allier, 2016 : s. p.).

5 Le fait de mêler informations d'ordre scientifique, intentions pédagogiques et mise en scène spectaculaire n'est toutefois pas radicalement nouveau en 1878. Depuis près d'un siècle, et l'invention du panorama par Robert Barker en 1787, différents entrepreneurs privés, intellectuels, architectes et artistes se sont demandé comment lier l'utile à l'agréable en faisant de ce lieu de spectacle qu'est le panorama un espace qui soit aussi dédié à l'enseignement populaire (Besse, 2003 ; Thomine-Berrada, 2015 : 30-35). La convergence et la familiarité entre panorama et diorama ne sont jamais aussi grandes que lorsque, comme dans le panorama Bourbaki, inauguré en 1881 et aujourd'hui exposé à Lucerne, des objets réels viennent animer le faux terrain en avant de la peinture monumentale circulaire, confortant l'illusion d'un spectacle (Finck \& Ganz, 2000). Ce jeu d'illusion pourrait être considéré comme l'héritage de l'art baroque italien, qui mêle également peinture en trompe-l'œil, sculpture et architecture (Blanc, 2015 : 106-113). La première question que posent conjointement panoramas et dioramas touche donc à la limite entre le vrai et le faux, l'objet authentique et le décor créé autour de lui. Dans le cas du panorama, l'objet réel est un accessoire de la représentation générale ; dans le diorama, c'est l’inverse : le décor est utile à la mise en scène de l'objet.

$6 \quad$ La présence de mannequins dans les premiers dioramas du MET (figure 1) autorise un autre parallélisme avec les musées de personnages en cire, inaugurés dès la fin du XVIIIe siècle par Madame Tussaud, d'abord en France puis en Angleterre, après la Révolution. Le musée permanent de Madame Tussaud est ouvert à Londres en 1835, bien avant la vague de créations de musées de cire en Europe, des années 1880-1890 (Sandberg, 2002 : 18-26). Géographiquement et chronologiquement le plus proche du MET, le musée Grévin est inauguré à Paris en 1882, avec un certain nombre de scènes historiques reconstituées, un soin attentif porté à la qualité des décors et le même sensationnalisme de la vraisemblance mise en ouvre avec soin (Schwartz, 1995, 2017 : 50-52 ; Martinez, 2017).

Figure 1. Section française du Musée d'Ethnographie du Trocadéro, Scène d'intérieur : mannequins en costume de Bretagne, Paris, 1884-1928, photographie anonyme, ph.1962.205.3 


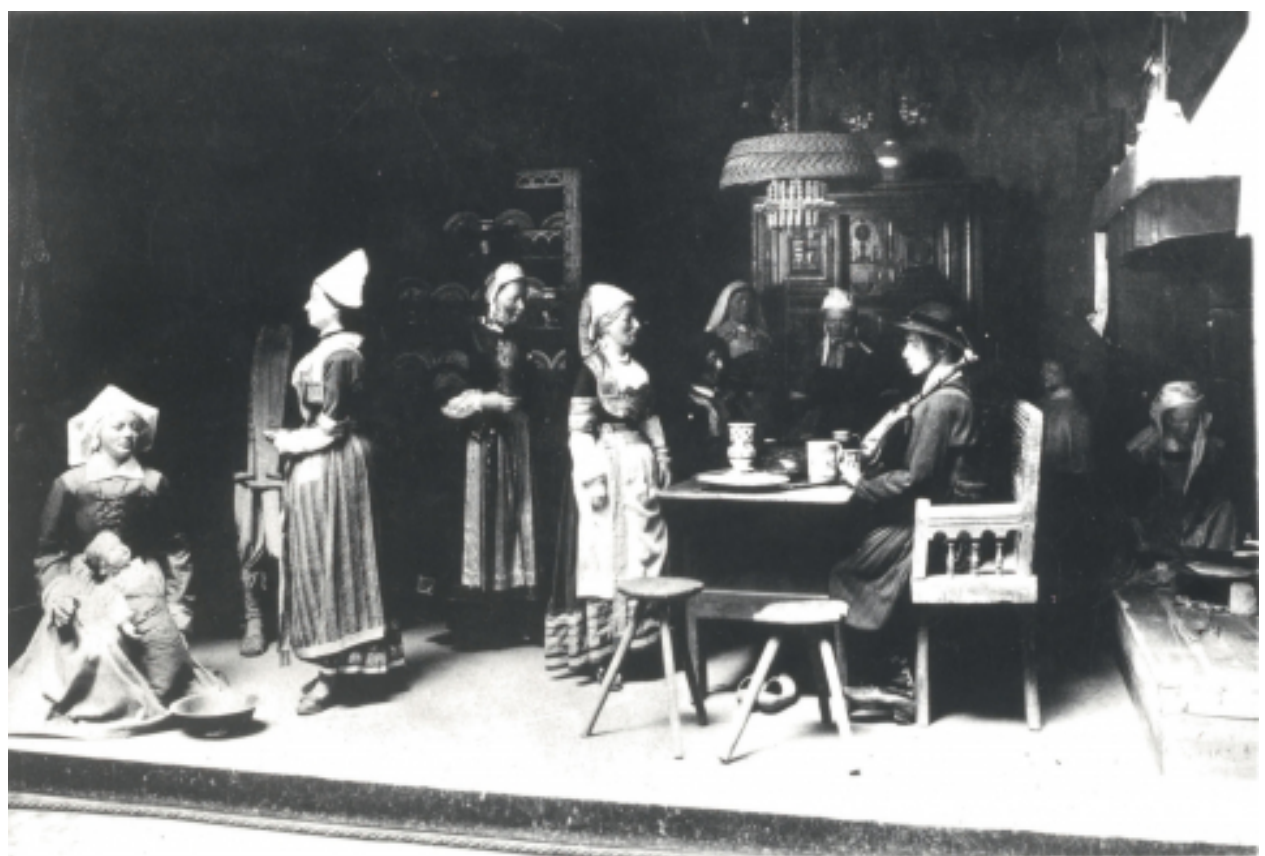

(C) Mucem

Pour cette première période allant de 1878 à 1928, les Expositions universelles, les panoramas et les musées de cire partagent un certain nombre d'enjeux (politiques, esthétiques, pédagogiques, éducatifs...) avec les dioramas de la salle de France, inaugurés en 1884 au sein du musée d’Ethnographie du Trocadéro. Ils ont pu leur servir de source d'inspiration plus ou moins directe ou de prolongement. L'invention du cinéma dans les dernières années du XIXe siècle et l'obsolescence progressive des panoramas qui en découle sont contemporaines du sentiment de désuétude que suscitent peu à peu les premiers dioramas du MET. La décision de les supprimer est liée au rejet plus général de la muséographie de la salle de France de 1884 . sa nomination à la direction du MET en 1928, Paul Rivet ferme la salle de France et nomme l'année suivante Georges-Henri Rivière comme adjoint : autant de signaux qui témoignent d'une volonté de rupture franche. Les années 1920 et 1930 sont en effet marquées en France par un contexte général de rénovation des musées et de la muséographie : on passe d'un goût marqué par l'accumulation d'objets réunis dans un dispositif touffu à une esthétique épurée, dont l'ordonnancement est dicté par les connaissances scientifiques les plus actualisées. Le Bulletin des musées de France se fait l'écho de cette mutation avec des doubles pages à but démonstratif, confrontant l'état de la muséographie avant et après rénovation (Anonyme, 1934 : 24-30). Porté par ce mouvement général, le MET accomplit lui aussi sa mue.

8 Dans le journal Comodia du 19 juin 1932, un article sur l'exposition des bronzes et ivoires du Bénin démontre que Rivière a su rapidement créer des liens entre le MET et les artistes de son temps, faisant entrer le musée du Trocadéro de plain-pied dans la modernité. Intitulé « De M. de Monzie à M. Matisse, tout Paris a défilé au Trocadéro », ce texte indique comment il a su transformer en profondeur le MET « qui était, il y a peu d'années encore, un tombeau » (Anonyme, $1932: 3$ ). Il est donc essentiel de comprendre en quoi la muséographie de Rivière apparaît alors si innovante, si moderne, et en opposition avec la majorité des pratiques de l'époque.

$9 \quad$ Inspirée par ses voyages, la perspective de Rivière se distingue d'autres visions du musée plus marquées par une approche propre aux beaux-arts. Dans les années 1930, les débats dans le monde des musées sont vifs, et les prises de positions contradictoires se succèdent. Un article intitulé « Musées pour le peuple », du 28 octobre 1936, en témoigne de manière aiguë tout en révélant les prémices de ce qui va devenir l'unité écologique :

\footnotetext{
« Deux thèses s'affrontaient, [...] Rivière, les yeux remplis de ce qu'un récent voyage en Russie lui avait "révélé" disait-il, exposa la première. C'est la présentation en quelque sorte matérialiste des collections dont se compose le musée, présentation qui cherche à mettre l'accent spécialement sur les “conditions" de la production des œuvres, c'est la mise en valeur des vieux facteurs
} 
historiques déterminants de Taine, le milieu, la race et le moment. Pour

"expliquer" les tableaux des Le Nain par exemple, on les environnera de

documents se rapportant à la vie paysanne au XVIIe siècle. Pour "expliquer"

[l']Art romantique, on le fera précéder de documents sur les Guerres du Premier

Empire, illustrant un passage célèbre de la Confession d'un enfant du siècle. Ainsi,

concluait le conférencier, le musée paraîtra plus "dynamique", plus "vivant". Il

sera également plus accessible au public moyen.

L'autre thèse, que nous pourrions appeler "le point de vue de la sensibilité", fut

défendue brillamment par les conservateurs du Louvre» (Lemoine, 1936 : 5).

10 Dans les années 1940 et 1950, le discours de Rivière se précise pour définir des environnements muséographiques comme des «tranches de réalités » (Bouiller \& Calafat, 2017a : 210-214). Ils apparaissent en contrepoint d'autres propositions muséographiques plus systématiques : « Des méthodes d'exposition s'expérimentent et s'élaborent, qui vont de l'exposition systématique par techniques de fabrication, fonctions techniques, économiques, sociales, idéologiques, styles et motifs artistiques, époques, complexes culturels, etc., jusqu'à la présentation de tranches de réalités » (Rivière, 1946 : 26). L'enjeu est d'apporter dans l'enceinte du musée, par le truchement de ces dernières, des exemples caractéristiques d'un lieu, d'un moment et d'un milieu social, puis de les accompagner par la documentation nécessaire. Il est précisé en note de bas de page qu'il s'agit de « l'équivalent des restitutions établies par Vergnet-Ruiz et l'architecte M. Bitterlin avec la plus grande rigueur scientifique, à l'aide des anciens inventaires, dans les appartements royaux et impériaux de Compiègne » (ibid. : 26)².

Dans le Bulletin des musées de France, Jean Vergnet-Ruiz est l'auteur d'un article sur le château de Compiègne, sous-titré : " Essai d'une doctrine de restitution des appartements historiques ». En 1946, ce travail est encore unique en son genre :

«La conservation du musée national de Compiègne vient d'entreprendre un
important travail scientifique ; il constitue le premier essai en France de
restitution historique totale du cadre de la vie des anciens souverains dans les
parties ouvertes au public d'une demeure de la Couronne, en fonction d'un décor
mural subsistant pour chaque salle, des événements historiques qui s'y
déroulèrent ou des possibilités mobilières » (Vergnet-Ruiz, 1946 : 15).

Il précise qu'il s'agit d' « une restitution historique totale des grands appartements en instaurant une discipline scientifique rigoureuse qui ne parait jamais avoir été systématiquement appliquée en France ni à l'étranger ». Dans l'article, Vergnet-Ruiz précise la méthodologie adoptée : "n'exposer dans une salle déterminée que les objets, meubles, tentures, etc., ayant figuré ensemble à une date donnée dans cette salle, à l'exclusion d'aucun autre objet, quel qu'en puisse être l'intérêt ou la beauté, même s'il a figuré dans cette même salle à une autre date "(ibid.). Cette proposition muséographique possède une visée aussi immersive que les unités écologiques. Elle propose aux visiteurs une approche pédagogique et concrète, leur offrant presque l'illusion de se déplacer dans le temps, du fait de son souci scientifique à contextualiser et à historiciser. Vergnet-Ruiz insiste ainsi sur la valeur exemplaire de cette proposition :

\footnotetext{
« L'intérêt historique et très souvent la beauté des restitutions, où le rapport des formes, des matières et des couleurs apparaît comme une précieuse leçon de goût des anciens (certains ensembles étonnent, d'autres sont d'une qualité presque émouvante), doivent forcément emporter les hésitations et lever les obstacles » (ibid.).
}

Les éléments de comparaison avec les unités écologiques sont nombreux : volonté de renouvellement muséographique, démarche scientifique rigoureuse strictement revendiquée, exemplarité pédagogique définie comme un but à atteindre, dimension spectaculaire et séduisante parfaitement assumée. Pour Rivière, les unités écologiques, ensembles concrets, réels, tirés de la vraie vie, répondent à ces quatre objectifs. Elles possèdent bien sûr une valeur éducative, mais sont également défendues comme étant des éléments attrayants du futur musée 3 .

14 La démarche de collecte qui débouche sur la création des unités écologiques se met en place petit à petit après la Deuxième Guerre mondiale (Bouiller \& Calafat, 2017a : 
212). La première, celle du tourneur sur bois, est acquise en 1965. Outre le modèle historique de Compiègne, la méthodologie s'apparente délibérément à celle de l'archéologue. Afin de donner un caractère scientifique à la collecte, Rivière insiste sur la phase de prélèvement des objets authentiques, au sein d'un milieu, dans leur écologie :
« À l'exemple de l'archéologue, nous fouillons un terrain : un terrain social, qui a lui aussi ses gisements et sa stratigraphie, dont l'exploitation savante exige prélèvements de contexte, analyses, patientes notations. Éclairant les apports de la découverte, toute une documentation est à produire par le chercheur et ses techniciens : journal d'opération, inventaires, fiches, relevés, photos et films de l'inanimé, dont nous partageons les méthodes avec l'archéologue, photos, films, phonogrammes du vif, propres à l'ethnologue4. »

Ce recours appuyé au vocabulaire scientifique de l'archéologie, les profils de chercheurs et de techniciens mis en avant, l'arsenal d'outils documentaires, traduisent un certain rapport à la réalité qui ne laisse guère de place à l'approximatif ou à l'improvisation. Inspirée des travaux d'André Leroi-Gourhan sur le site de Pincevent, l'unité écologique témoigne de la volonté de Georges-Henri Rivière d'expliciter les objets dans leurs relations avec les hommes et leur milieu naturel. Son objectif est de donner à voir les objets dans leur contexte, ce qui nécessite un exercice préalable rigoureux in situ, proche de la fouille en archéologie. En 1973, Georges-Henri Rivière en arrive ainsi à cette définition :

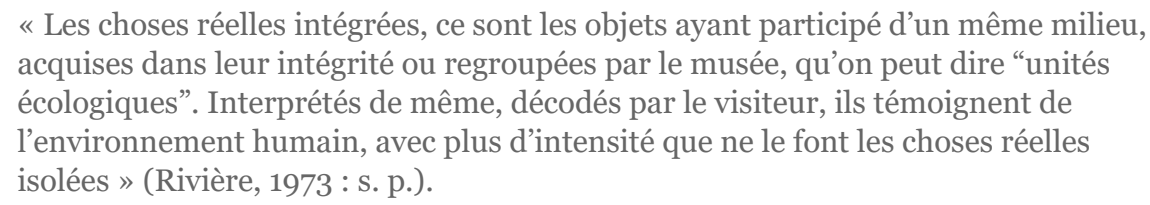

Pour Compiègne comme pour l'archéologue, c'est la réalité historique, les documents d'archives, les objets authentiques collectés et les faits du terrain qui dictent sa conduite au chercheur, devenu muséographe. C'est donc sur cette notion même de réalité, telle qu'elle est modélisée par les différents types de dioramas, qu'il faut se concentrer.

\section{L'authentique et le vraisemblable}

La réception des dioramas de la salle de France du MET, ouverte en 1884, offre un témoignage qui va dans le sens de la puissance du jeu de vraisemblance, très à la mode dans cette décennie 1880 (Papet, 2018) :

\footnotetext{
«Dans une grande salle bien éclairée, quelques femmes bretonnes et frisonnes, et un intérieur breton de grandeur naturelle, frappant de vérité. Tout y est, pots, lits en forme d'armoires de bois ouvragé, et le vieux grand-père, toujours gelé, assis dans l'âtre même du foyer. Ce décor, très bien réglé, a le don d'attirer la foule » (Lami, $1886: 727$ ).
}

On comprend entre les lignes que le caractère scientifique de la proposition muséographique n'est pas particulièrement mis en avant. Ce qui est remarquable, c'est avant tout l'aspect pittoresque d'un patrimoine qui est alors déjà considéré comme un héritage en perdition (DeGroff, 2012 : 126-129).

Le goût pour la reconstitution de la réalité est accentué par la présence de mannequins en costumes qui animent et rendent attrayantes les scènes. Les personnages sont représentés non seulement par leur costume, mais aussi par leur " type » physique, selon les travaux d'anthropologie physique alors en plein développement (figure 2). Les têtes des mannequins sont bien moulées " sur nature ", comme nous l'apprennent les devis des entreprises commanditées 5 . Le souci du détail et du sensationnel est poussé très loin grâce au choix de ces entreprises, qui appartiennent pour certaines au monde du spectacle vivant (comme, par exemple, le perruquier Dieudonné, par ailleurs fournisseur de la Comédie-Française), davantage qu'à celui de la sculpture polychrome, pourtant en plein développement dans cette 
décennie. On perçoit ici, sous-jacente, la tension habituelle autour des questions de muséologie, oscillant entre scientificité et spectaculaire, l'éducation du citoyen demeurant la finalité de cet équilibre à trouver entre l'un et l'autre.

\section{Figure 2. Tête de mannequin de la section française du Musée d'Ethnographie du} Trocadéro, Paris, vers 1884, Mucem, 2018.41.2

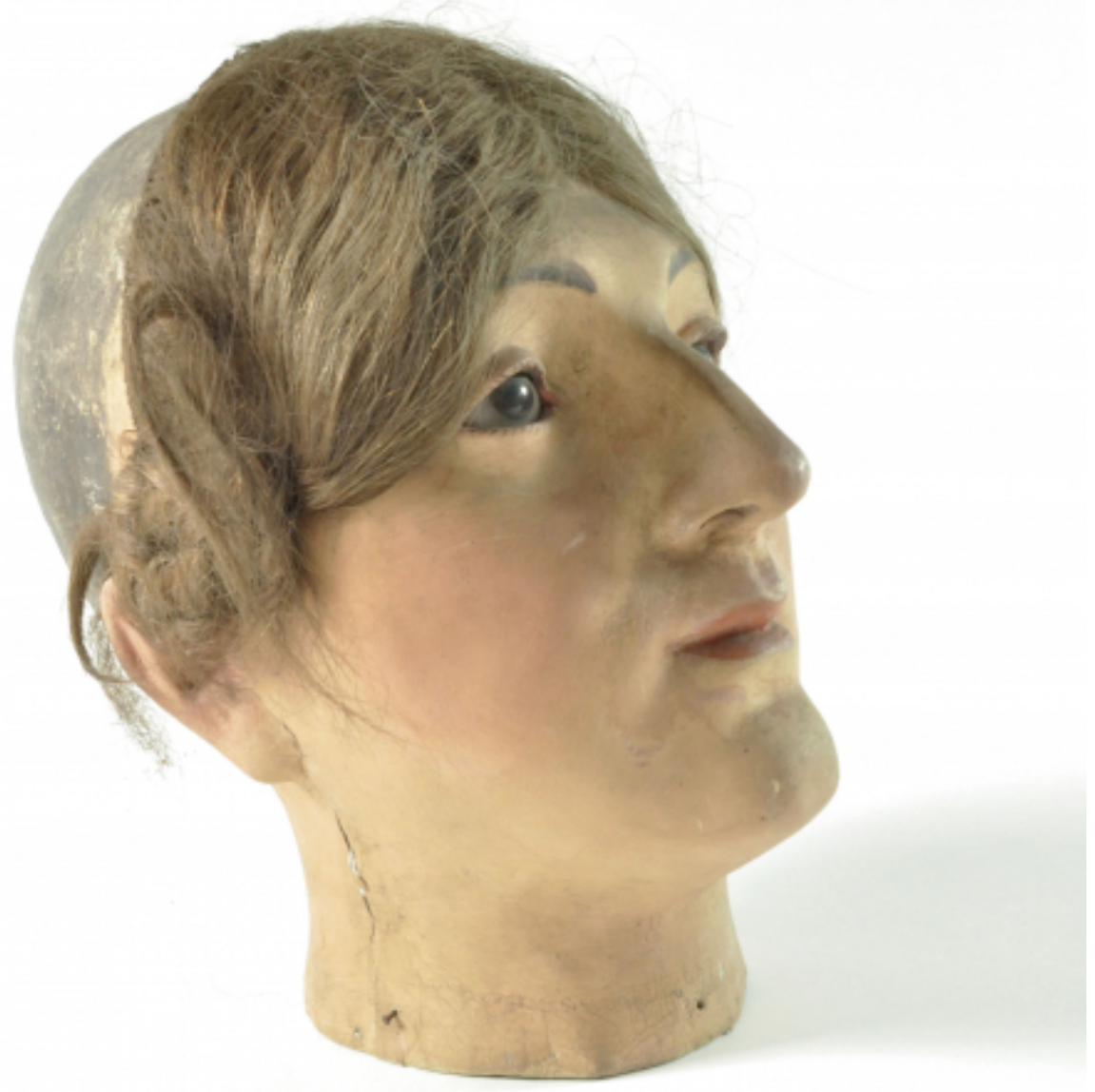

(C) Mucem

Si les outils et subterfuges du monde du spectacle vivant sont exploités par les muséographes, ils demeurent clairement, dans l'esprit de leurs utilisateurs, comme des outils au service de la science et de la diffusion de cette dernière. Les archives conservées sur le projet de musée sont éclairantes à ce sujet, et pour comprendre la vocation que le MET s'assigne dès sa création. Le docteur Ernest Hamy s'adresse ainsi au ministre de l'Instruction publique :

\footnotetext{
« Les services qu'est appelé à rendre le musée spécial dont la création est sollicitée à Paris depuis près d'un siècle sont de divers ordres. En effet, les collections ethnographiques ne sont point seulement utiles à la connaissance de l'anthropologie [...] elles contribuent en outre dans une large mesure aux progrès des autres sciences naturelles et sont appelées à fournir des renseignements parfois fort précieux aux économistes, aux commerçants, aux industriels, aux artistes 6 . »
}

Comme pour certains panoramas à vocation pédagogique, le but affiché d'une éducation citoyenne et populaire demeure la finalité ultime du projet muséographique. Il est intéressant de souligner que le musée est revendiqué comme n'étant " point seulement » un outil scientifique. Ce fait peut expliquer les compromis voire les compromissions des muséographes avec les divers outils du monde du spectacle. Tout se passe comme si les accommodements avec la réalité (comme la création de mannequins illusionnistes) étaient acceptables pour autant que les subterfuges cherchent au maximum à faire oublier qu'ils en sont (par exemple en s'appuyant sur la méthodologie de l'anthropologie physique pour mouler sur nature les visages des 
mannequins), et pour autant que leur but demeure de rendre accessible une connaissance. La transmission du savoir à un large auditoire prend la priorité sur l'accumulation ou la préservation du savoir lui-même.

Les Expositions universelles de Paris de 1855, 1867, 1878 et 1900 manifestent également cette volonté de mise en valeur spectaculaire. Comme on l'a vu, elles peuvent être considérées comme la principale source d'inspiration des dioramas du MET grâce aux véritables préparatifs et aux expérimentations concrètes auxquels elles donnent lieu (voir note 1). Mais elles peuvent apparaître aussi comme des prolongements de ce qui est proposé dans la salle de France, comme en témoignent par exemple les échanges de correspondance entre les ministères à l'occasion de l'Exposition de 1900.

23 Cette année-là, Jules Sain, délégué du ministère de l'Agriculture, rédige le Musée rétrospectif du groupe VII - Agriculture - à l'Exposition universelle internationale de 1900 à Paris. La muséologie proposée ici semble correspondre à une immersion dans les provinces françaises, dans une vision homogénéisante du monde rural. Il s'agit en effet d'englober toute la paysannerie et ses différents domaines : " Les objets se rapportent à l'agriculture générale, à la viticulture et à l'œnologie, aux cultures diverses, à l'élevage, à la laiterie et à la fromagerie, aux industries agricoles, etc. » En outre, Jules Sain décline tout un champ lexical autour de la curiosité et de l'attractivité :

« Les objets [...] étaient exposés dans de pittoresques bâtiments de ferme, reconstitués d'après d'anciens types curieux de diverses régions de France, appropriés à chaque genre et artistement entourés de feuillage et de verdure. Cette sorte de petit village était animé par des groupes de mannequins vêtus des costumes des provinces françaises et représentant les diverses scènes de la vie des champs dans l'emploi usuel de l'outillage ancien » (Sain, 1903:5).

Il ajoute :

« C'était ici, sous un vieux hangar à la charpente vermoulue reposant sur de rustiques piliers de pierre, un gamin d'une douzaine d'années venant quérir au milieu d'une quantité de charrues, de herses, etc., de toutes les contrées de France, un semoir à brouette d'un singulier modèle, pendant qu'un paysan grimpé sur une échelle décrochait du plancher où ils étaient pendus en grand nombre et fort variés les outils dont il avait besoin » (ibid.).

Ce caractère vivant est ce qui manque aux dioramas du MET, mais on saisit ici le glissement entre les mannequins évoqués et les acteurs réels employés temporairement pour l'Exposition universelle. On perçoit également les liens qui existent entre dioramas et exhibitions d'êtres humains vivants telles qu'elles ont pu se développer aux XIXe et XXe siècles (Snoep et al., 2011 ; Bouiller \& Calafat, 2017b : 41 ; Étienne, 2017 : 186-193). La portée politique des propos tenus, des discours et des projets développés à l'occasion de ces Expositions universelles apparait ainsi de manière assez évidente et peut aussi valoir pour les dioramas. La réalité ainsi représentée revêt une dimension figée et fantasmatique, elle-même en contradiction avec le monde réel. Si le diorama cherche à fixer le réel, ce dernier est au contraire toujours en mouvement. S'il en donne une image fantasmée (propre, claire et nette, sans nuance possible, sans trace de mélange, ne souffrant pas l'exception qui confirme la règle, allant jusqu'à chercher des acteurs qui jouent leur propre rôle dans les décors reconstitués des Expositions universelles), cette dernière ne permet jamais, en définitive, de rendre compte de la complexité et de l'étendue de la réalité. Cette disruption avec le monde réel explique d'ailleurs sans doute en partie la propension du diorama à apparaitre plus ou moins rapidement suranné.

26 Lorsqu'en 1936 Rivière défend son projet d'un musée français des Arts et Traditions populaires lors d'un cours de muséologie à l'École du Louvre, il l'associe à la question des musées de plein air et insiste à nouveau lui aussi sur une approche globale de la réalité. Les exemples des musées au Danemark, en Suède et en Norvège alimentent sa réflexion. Le cas de Skansen, créé par Artur Hazelius, est cité pour « nous faire étudier par les yeux la vie et la civilisation des temps anciens. Les objets ne sont pas [...] isolés, comme dans une collection scientifique, mais ils sont groupés de manière à former un tableau général. Nous voyons la chambre entière avec son mobilier et tout son contenu » (Rivière, $1936: 63$ ). 
Dans ces propos, la notion de " tableau général » semble être assez proche de certaines propositions d'acquisitions du MET, assez rares pour être soulignées. Le premier exemple dans les dossiers d'œuvres du MET date de 1907, lorsque Mme Landrin lance une enquête sur l'assistance publique : elle parle déjà de " tableaux ». Tels et tels objets sont à collecter car ils pourront prendre place dans un discours qui passera par une succession de tableaux ${ }^{7}$. Le modèle de l'enquête est nouveau en 1907 et très idéologique. Ce n'est pas l'intérêt pour le contexte qui est manifesté ici, mais pour le discours que l'objet peut permettre de véhiculer, par exemple ici le quatrième et dernier tableau : "Les enfants abandonnés recueillis par la République élevés à ses frais, sont enrôlés pour la défense de la Patrie. » Si Rivière innove en matière de muséographie par rapport au MET, ce n'est pas tant sur ce point de l'idéologie du contexte que sur la méthode destinée à produire les nouvelles offres muséographiques. Cette méthode fait une large place à des savoir-faire spécifiques exogènes au monde des musées (architectes, décorateurs...) et à une approche scientifique pluridisciplinaire (géographes, archéologues...).

Quand en 1939, l'ethnologie est à l'honneur au pavillon français de l'Exposition universelle de New York, Rivière s'associe à l'architecte Guy Pison (figure 3). Afin de reconstituer des intérieurs français typiques (arlésien ou breton, par exemple), la combinaison de l'ethnologue-conservateur et de l'architecte semble nouvelle et promise à un avenir que la muséologie actuelle n'a pas désavoué. Cette exposition de 1939 fait d'ailleurs également la part belle à l'architecture vernaculaire, avant même les chantiers intellectuels de l'« Enquête sur l'architecture rurale » (EAR), menés en France dans les années 1940. Les maquettes sont un modèle de présentation presque indispensable pour donner à voir en trois dimensions ce patrimoine bâti dans une exposition. Les savoir-faire d'architecte d'intérieur ou de scénographe, très tôt compris comme nécessaires par Rivière, sont ainsi parmi les premiers à être mobilisés.

Figure 3. Pavillon français, section du tourisme : le Musée paysan. Intérieur de la BasseBretagne présenté avec le concours du Musée des Arts décoratifs de Nantes lors de l'Exposition internationale et universelle de New-York en 1939, photographie de John Towse, ph.1941.18.14

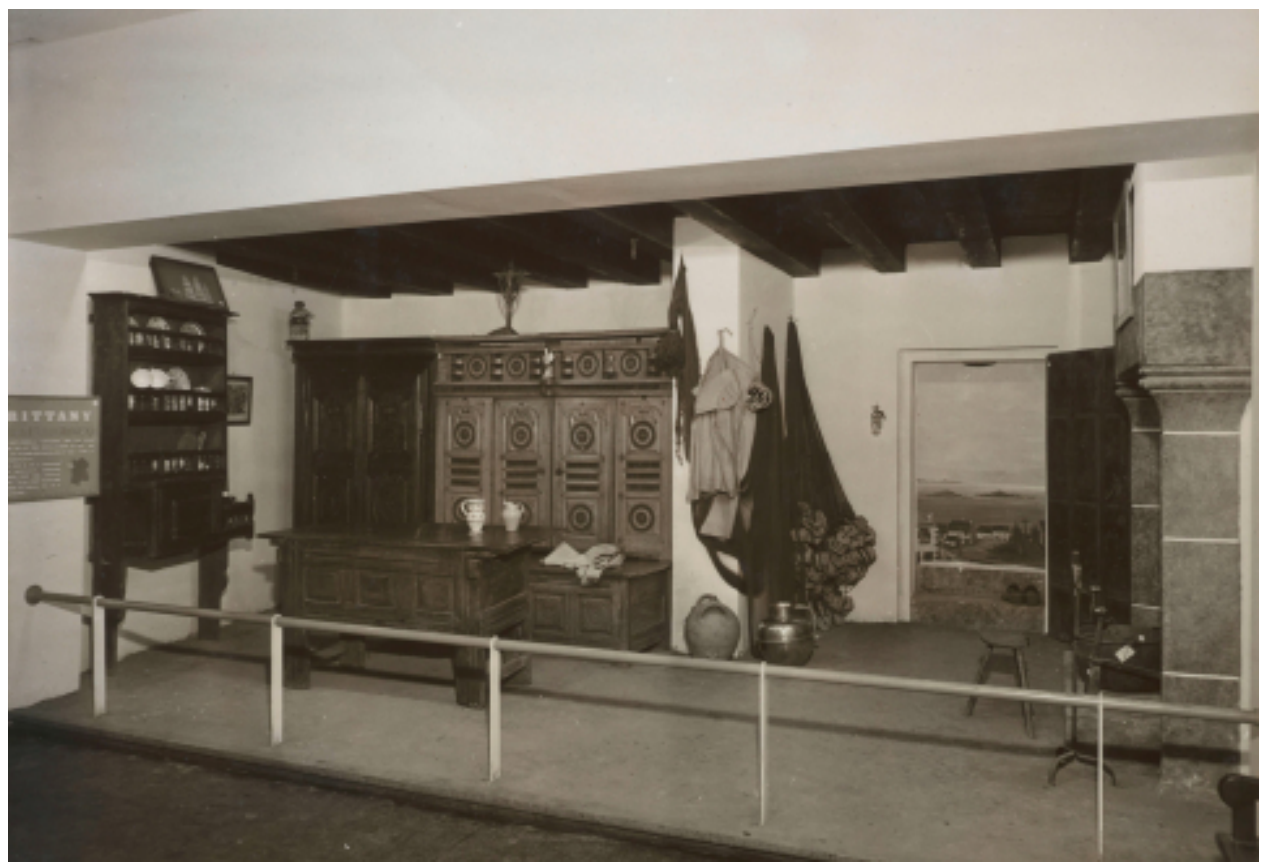

(c) Mucem

Quant au métier de peintre-décorateur, indispensable pour la mise en œuvre concrète des unités écologiques, il semble qu'il soit encore pleinement d'actualité dans ces années 1930, comme en témoigne cette incise d'Arsène Alexandre, soulignant l'entremêlement des vertus décoratives et pédagogiques, documentaires et enchanteresses, des grandes peintures réalisées dans l'esprit de reconstitution : 
À la différence des dioramas de la salle de France au MET, les unités écologiques de la galerie culturelle du MNATP ne sont pas des modélisations ou des projections de l'image que l'on peut se faire de tel ou tel lieu, telle ou telle entité culturelle. Elles sont au contraire définies dans leur hyper-réalité propre et concrète (Cuisenier, 1987 ; Desvallées, 1992, 2009 ; Rolland-Villemot, 2016 ; Tamarozzi, 2017). La documentation qui les accompagne (carte géographique, relevés, photographies, entretiens...) témoigne de cet ancrage dans le réel le plus identifié et le moins fantasmatique possible. Ce sont des « spécimens issus de la nature et des objets issus de l'homme ${ }^{8}$ ».

Dans les faits, le principe de reconstitutions intégrales demeure un leurre. Ces objets réels prélevés dans la vraie vie deviennent et demeurent des objets appartenant au langage du musée, avec toutes les problématiques pratiques qu'ils posent : accumulation d'un grand nombre d'items, conservation de matériaux fragiles, précautions à prendre pour leur installation... L'unité écologique reste également un médium spécifique dans l'exposition, avec toutes les contraintes de cette dernière : nécessité de ménager une grande baie dans un mur pour permettre aux visiteurs d'embrasser l'intégralité d'une pièce, nécessité de reconstituer certains éléments architecturaux non transportables, impossibilité de conserver les matières périssables (pain, fromage... qui vont devoir être reconstitués en matériaux pérennes).

De nouveau se pose la question de la limite entre le vrai, le décor reconstitué, le faux vraisemblable ajouté au réel (par exemple un fer peint imitant le fer rougeoyant dans la forge du forgeron). La rigueur scientifique ne permet pas d'être absolument fidèle au principe d'une réalité exactement déplacée dans le musée. Le simple phénomène du déplacement est lui-même antinaturel et s'ajoute aux hasards de l'enquête, à l'arbitraire du choix du spécimen prélevé, au caractère figé dans le temps (figure 4).

Figure 4. Intérieur de la salle principale d'une ferme photographié, enquête en BasseBretagne du MnATP, 21 juillet 1939, Chez les Kintrek à Surzur, photographie de Jeannine Auboyer, ph.1940.2.94 


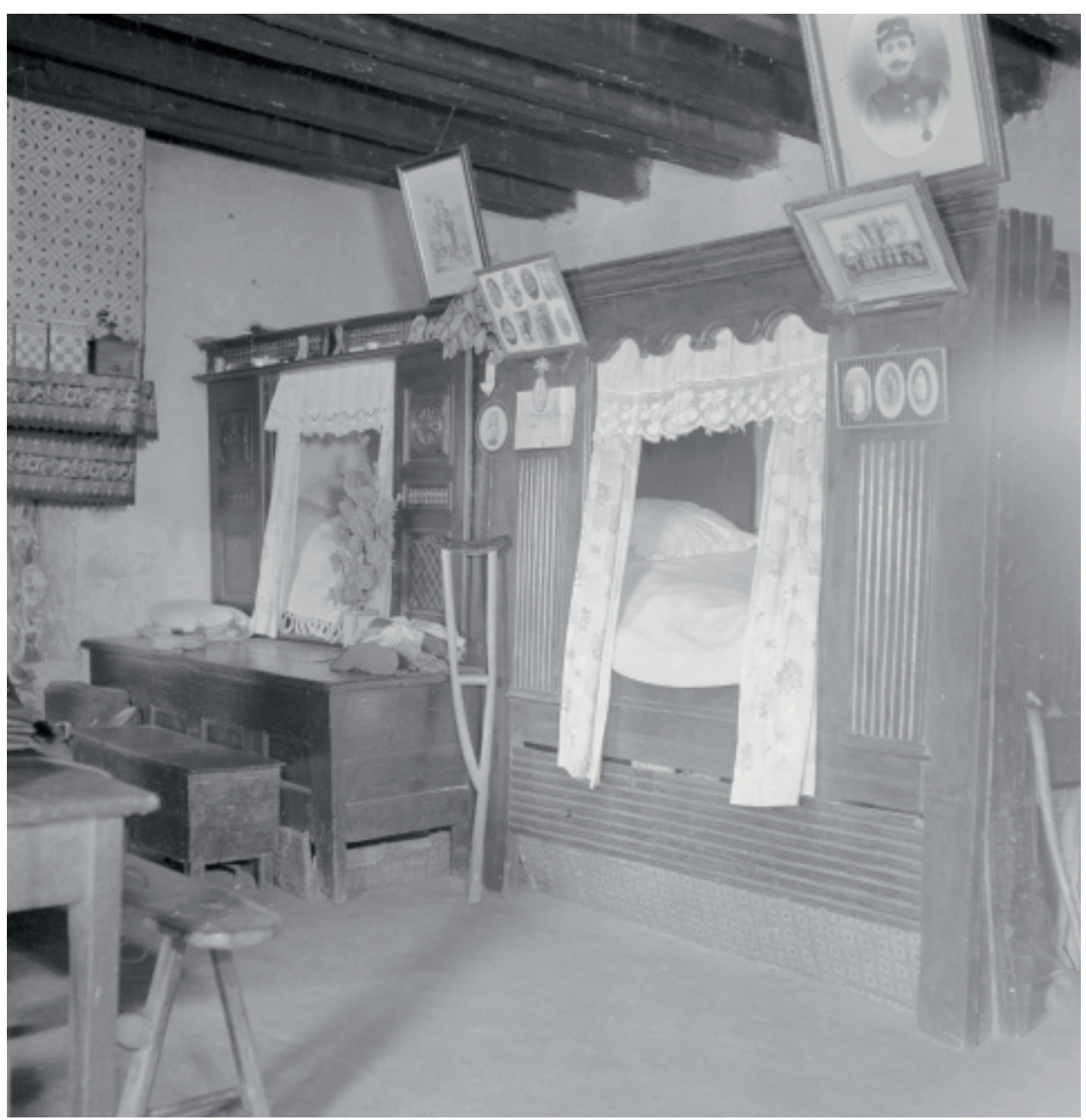

(c) Mucem

La force de l'unité écologique ne réside donc sans doute pas dans les « progrès » que sa méthode de création lui a permis de réaliser par rapport aux premiers dioramas du MET. En revanche, elle pousse très loin la réflexion sur le développement d'une méthodologie rigoureusement scientifique qui prend en compte l'influence de l'observateur sur le terrain observé ; sur les techniques modernes de prélèvement « objectif » d'un fragment d'hyper-réalité ; sur les techniques modernes de restitution de cette réalité au sein du musée (jeu d'éclairage, spectacle son et lumière...) ; sur l'expérimentation de la pluridisciplinarité pour aborder des sujets aussi complexes (figure 5). Le fait même que le déplacement de la réalité puisse apparaître comme un leurre n'est peut-être pas dénué d'intérêt. C'est exactement dans les mêmes années que beaucoup d'artistes contemporains développent une réflexion approfondie sur les liens entre l'art et la rue, sur le glissement ténu entre le réel et l'artefact, sur l'art comme expérience vécue, et l'œuvre comme truchement d'un certain rapport à la réalité.

Figure 5. Unité écologique de la salle commune de la maison de Catherine et Guillaume Deuffic vers 1939, Goulien, Finistère, vue de la galerie culturelle du MNATP, 2005, photographie de Danièle Adam, php.2005.3.280 


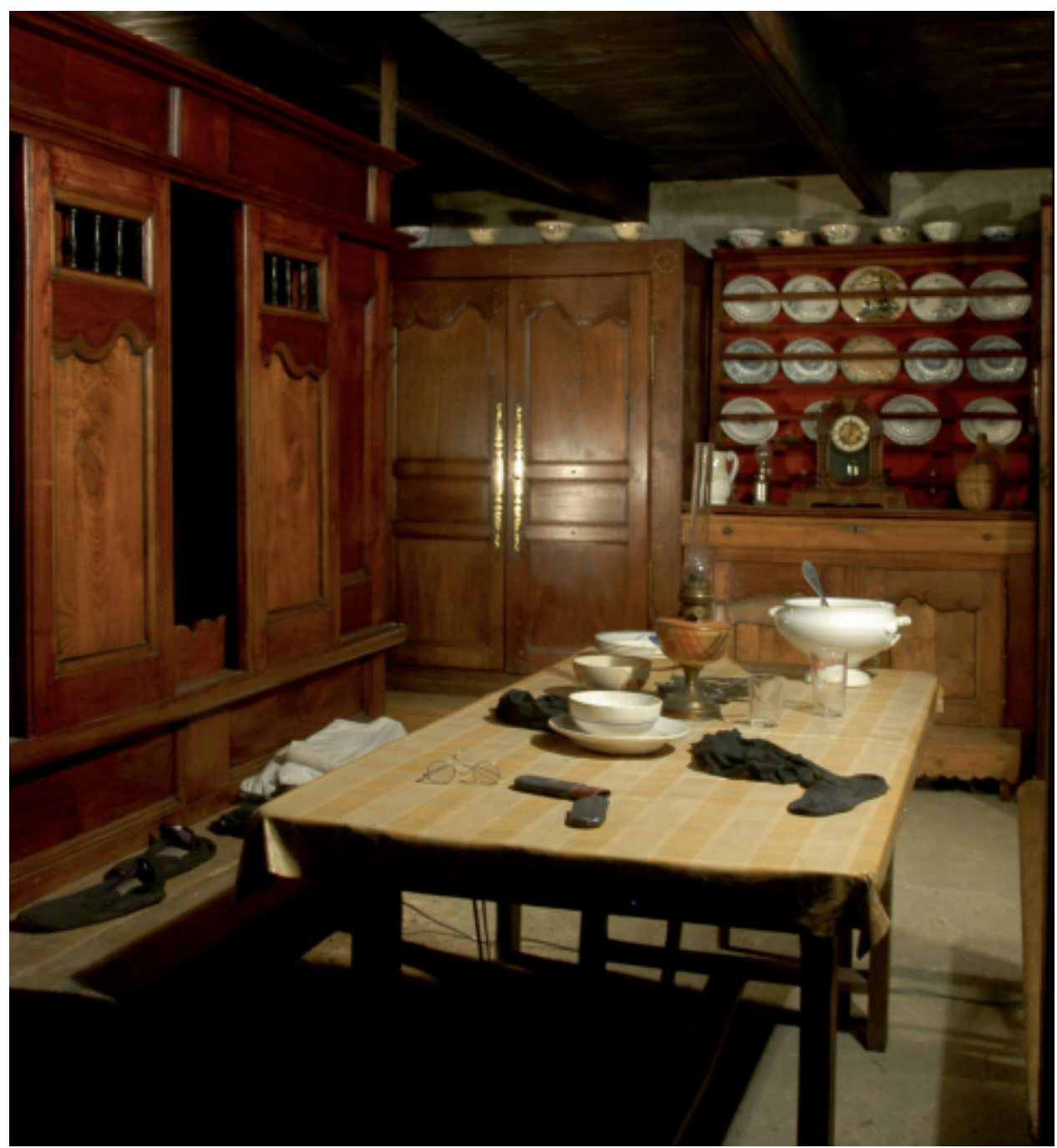

(c) Mucem

\section{Les ateliers d'artistes : une nouvelle forme de dioramas?}

Après avoir évoqué la genèse des unités écologiques entre 1937 et 1975, et tenté de comprendre leurs liens avec l'émergence simultanée de la notion d'installation dans le champ de l'art contemporain (Bouiller \& Calafat, 2017b : 36-55), nous proposons de les voir avant tout comme un phénomène éminemment de leur temps. Le jeu d'ambiguïté qu'elles entretiennent avec la réalité, le rôle d'attraction spectaculaire qu'elles assument parfaitement, autorisent à chercher leurs reflets conceptuels et matériels dans quatre exemples de dioramas des mêmes années, mais appartenant au champ des musées et galeries d'art contemporain.

En 1957, à sa mort, Constantin Brancusi lègue à l'État français la totalité de son atelier avec tout son contenu, sous réserve que le musée national d'Art moderne s'engage à le reconstituer tel quel. Une première restitution partielle est réalisée au sein de ce dernier au Palais de Tokyo en 1962. Une reconstitution intégrale est ensuite présentée entre 1977 et 1990 à proximité du Centre Pompidou, avant que Renzo Piano ne conçoive une architecture spécifique pour lui en 1997, incluant des espaces de circulation spécifiques pour les visiteurs (Tabart, 1997). Durant quarante ans, les vicissitudes liées à la complexité de la conservation et de la présentation au public de cet atelier "dans son jus » témoignent de la difficulté pour une institution muséale d'accompagner la transformation d'un ensemble complexe, depuis son statut d'objet réel à celui d'objet muséifié. Mais cette expérience fondatrice est peut-être une de celles qui ont contribué à faire basculer les problématiques de l'unité écologique du champ des musées d'ethnographie à ceux des musées d'art, soulignant l'importance du in situ, 
de l'idée d'œuvre d'art totale ou du dialogue entre les œuvres pour les artistes (Rodriguez, $2002:$ 132-138).

C'est en 1975, l'année même de l'inauguration des unités écologiques au MNATP, que le musée national d'Art moderne de Paris inscrit sur ses inventaires le Magasin de Ben, sous le $\mathrm{n}^{\circ} \mathrm{AM}$ 1975-185, deux ans avant sa présentation au public dans le tout nouveau Centre Pompidou. Il s'agit bien là aussi de prélever un morceau de réalité dans son entièreté, de la figer, de la déplacer vers le musée et de la restituer au public en donnant l'illusion d'une fidélité parfaite à l'original. Les mêmes outils méthodologiques que ceux de l'unité écologique sont utilisés : relevés, photos, inventaire précis, entretiens avec le créateur-utilisateur. La seule différence - de taille - réside dans le fait que ce dernier est un artiste de renommée internationale et que sa boutique, quoique réellement utilisée comme boutique pendant des années, prend immédiatement le statut d'œuvre d'art plutôt que de témoin d'une pratique commerciale ordinaire, dont elle n'est en effet pas représentative. Il reste toutefois saisissant de constater que la même année, deux dispositifs conceptuels et matériels similaires, donnant lieu à des processus de prélèvement dans la réalité très proches, entrent dans les collections des musées nationaux et que la seule chose qui les distingue concerne l'interprétation qu'on propose de la nature et du statut du fragment de réalité prélevé et présenté. Il en est même des problématiques de conservation, restauration et remontage qui se posent dans les années 2010 aux musées responsables de ces œuvres et qui présentent un certain nombre de convergences théoriques et pratiques9.

Autre exemple frappant et chronologiquement proche, l'exposition d'ouverture Paris / New York qui est inaugurée en même temps que le Centre Pompidou, à Paris, en 1977, se présente comme un parcours à travers des œuvres, certes, mais dans une muséographie originale. Comme au MNATP, l'architecture du musée est résolument moderne, mais Pontus-Hultén, le commissaire, n'en profite pas pour écrire l'exposition sous le signe du pur white cube. Les œuvres accrochées sur les cimaises sont ponctuées par des alcôves à l'ambiance non muséale, reconstituant le cabinet d'un collectionneur ou une célèbre galerie new-yorkaise. Alors même que tout un pan du champ artistique continue de revendiquer l'autonomie maximale de l'œuvre d'art et sa présentation dans un contexte le plus neutre possible, l'idée que l'œuvre puisse aussi s'apprécier dans son environnement " naturel », avec une certaine ambiance, en corrélation avec d'autres œuvres voire d'autres objets, fait une percée spectaculaire avec cette exposition (Pontus-Hultén, 1977).

38 Enfin, c'est en 1979 que l'artiste belge Guillaume Bijl réalise sa première installation selon un principe de tautologie avec la réalité. Il s'agit du déplacement d'une salle d'auto-école entière dans la galerie Ruimte Z, à Anvers, inaugurée le 20 avril 1979 et accompagnée d'un manifeste demandant l'abolition des centres d'art contemporain. L'artiste les juge en effet inutiles d'un point de vue économique et suggère leur transformation en espaces commerciaux. Le phénomène du déplacement de la réalité vers la sphère muséale est cette fois poussé à son comble. Comme la carte à l'échelle $1 / 1$ de Jorge Luis Borgès ${ }^{10}$, la réalité envahit ainsi potentiellement l'entièreté du champ artistique, et l'on ne peut guère aller plus avant dans l'illusion de la translation parfaite d'un fragment de réalité ; dans le jeu d'ambiguïté entre le réel, le faux et le vraisemblable ; dans la cristallisation du politique, du pédagogique et du scénographique.

\section{Conclusion}

Pour conclure, un siècle après l'ouverture du musée d'Ethnographie du Trocadéro, le concept et la forme matérielle du diorama se retrouvent bien ancrés dans le champ de la création artistique, en dépit ou grâce à leurs liens avec la réalité. Pour admettre ce grand écart, il faut regarder l'histoire des dioramas des musées nationaux français consacrés à l'ethnographie de la France comme un continuum sur le temps long.

40 Il faut aussi accepter de les mettre en perspective historique par rapport à leurs sources d'inspiration et à l'esprit de leur temps, depuis leur création au MET ; leur 
abandon en 1928 ; la construction, en réaction, de l'unité écologique par Rivière après 1937 ; sa concrétisation dans la galerie culturelle du MNATP en 1975, jusqu'à leurs ricochets dans l'art contemporain à la fin des années 1970.

Le fait de considérer les dioramas du MET et les unités écologiques du MNATP comme le fruit de trois ingrédients hétéroclites (contenu scientifique, idéologie politique, dispositif scénographique) permet de reconsidérer ces composantes non comme opposées mais comme intrinsèquement liées et complémentaires dans l'écriture muséographique. En adoptant ce point de vue, on comprend mieux les liens non revendiqués entre les dioramas du MET et les unités écologiques du MNATP, relevant pourtant d'époques, de logiques et d'intentions différentes. Leur raison d'être et leur forme sont pourtant bien les mêmes : il s'agit dans les deux cas d'un assemblage d'objets authentiques prélevés dans le monde réel, mis en scène dans un espace muséal fermé, offert à la vision des visiteurs, avec pour ambition de rendre compte d'un condensé de réalité.

Ce rapport à la réalité fait du diorama un instrument indissociable d'un certain positivisme. Il semble illustrer une forme de mirage, utopique et généreux : ses auteurs possèdent tous, à un moment, l'illusion d'être en mesure de percevoir la réalité de manière objective et de faire partager cette vision au plus grand nombre. Ils manifestent une foi dans la capacité de la réalité à se laisser capturer, résumer et réduire à une forme matérielle. Mais cette connaissance n'est pas pour eux quelque chose de pur, d'enfermé, d'intangible et de réservé. Avec le diorama, ils traduisent aussi la conviction que le savoir s'élabore, se construit et surtout n'existe pas en soi s'il n'est pas disséminé largement. Les dioramas apparaissent ainsi comme des outils de transmission grâce auxquels les muséographes partagent avec le public une représentation de la réalité que quelques chercheurs ont forgée d'abord pour eux, entre eux, mais avec la ferme volonté de s'en ouvrir à tous.

43 Paradoxalement, c'est grâce à sa dimension créatrice, artistique, que le diorama peut aujourd'hui le mieux échapper au sentiment de désuétude dans lequel il a été plongé. Sans vouloir opposer de manière factice l'objectivité scientifique à la subjectivité artistique, il nous semble que les dioramas du MET et surtout les unités écologiques du MNATP peuvent être reconsidérés comme des « installations » qui en disent autant sur l'état d'esprit de leurs créateurs, sur la modélisation de la réalité active à leur époque, sur le désir de partage des connaissances, que sur la réalité qu'ils entendent subsumer.

\section{Bibliographie}

Besse (Jean-Marc). 2003. Face au monde. Atlas, jardins, géoramas. Paris : Desclée de Brouwer.

Blanc (Jan). 2015. " "Une idée entièrement nouvelle" ? Le regard panoramique avant le panorama », p. 106-114 in J'aime les panoramas. S'approprier le monde / sous la direction de Laurence Madeline et Jean-Roch Bouiller. Paris : Flammarion.

Bouiller (Jean-Roch) \& Calafat (Marie-Charlotte). 2017a. « Les unités écologiques sont-elles des dioramas ? », p. 210-215 in Dioramas (catalogue d'exposition, Palais de Tokyo, 14 juin 10 septembre 2017) / sous la direction de Katarina Dohm, Claire Garnier, Laurent Le Bon et Florence Ostende. Paris : Palais de Tokyo et Flammarion.

Bouiller (Jean-Roch) \& Calafat (Marie-Charlotte). 2017b. « Les unités écologiques ou la vie mode d'emploi », p. 36-55 in Document bilingue. Réserves et collections : un autre Mucem / sous la direction de Érik Bullot et Sabrina Grassi. Marseille et Paris : Mucem et Manuella Éditions.

Cuisenier (Jean) (sous la direction de). 1987. Muséologie et ethnologie. Paris : Réunion des musées nationaux.

DeGroff (Daniel). 2012. " Ethnographic display and political narrative : the salle de France of the musée d'Ethnographie du Trocadéro », p. 113-135 in Folklore and Nationalism in Europe during the Long Nineteenth Century / sous la direction de Timothy Baycroft et David Hopkin. Leyde et Boston : Brill.

Desvallées (André). 1992. Vagues. Une anthologie de la nouvelle muséologie. Mâcon et Savignyle-Temple : Éditions W et MNES.

Desvallées (André). 2009. "L'expologie de Georges-Henri Rivière : des collectes systématiques aux unités écologiques. La question du contexte », p. 277-293 in Du folklore à l'ethnologie / sous la direction de Denis-Michel Boëll, Jacqueline Christophe et Régis Meyran. Paris : Éditions de la Maison des sciences de l'homme. 
Dias (Nélia). 1991. Le Musée d’Ethnographie du Trocadéro (1878-1908). Anthropologie et muséologie en France. Paris : Éditions du CNRS.

Dohm (Katarina), Le Bon (Laurent), Garnier (Claire) \& Ostende (Florence) (sous la direction de). 2017. Dioramas (catalogue d'exposition, Palais de Tokyo, 14 juin - 10 septembre 2017). Paris : Palais de Tokyo et Flammarion.

Étienne (Noémie). 2017. " La matérialité politique des dioramas », p. 186-193 in Dioramas (catalogue d'exposition, Palais de Tokyo, 14 juin - 10 septembre 2017) / sous la direction de Katarina Dohm, Claire Garnier, Laurent Le Bon et Florence Ostende. Paris : Palais de Tokyo et Flammarion.

Finck (Heinz Dieter) \& Ganz (Michael). 2000. Le Panorama Bourbaki. Zurich : Werd Verlag.

Martinez (Pascale). 2017. Le Temple et les Marchands - Une histoire du musée Grévin (18811921). Dijon : Les Presses du réel.

Papet (Édouard). 2018. En couleurs. La sculpture polychrome en France, 1850-1910. Paris : Hazan.

Pontus-Hultén (Carl Gunnar). 1977. Paris / New York. Paris : Centre Pompidou.

Rodriguez (Véronique). 2002. "L'atelier et l'exposition, deux espaces en tension entre l'origine et la diffusion de l'œuvre ». Sociologie et sociétés, 34(2), automne, p. 121-138.

DOI : 10.7202/o08135ar

Rolland-Villemot (Bénédicte). 2016. « Unités écologiques, period rooms : des ensembles d'objets mobiliers, de la collecte à la valorisation muséographique ». In Situ. Revue des patrimoines, 29, s. p.

DOI : $10.4000 /$ insitu.13373

Sandberg (Mark B.). 2002. Living Pictures, Missing Persons. Mannequins, Museums and Modernity. Princeton: Princeton University Press.

Schwartz (Vanessa). 1995. " Cinematic spectatorship before the apparatus: The public taste for reality in fin-de-siècle Paris ", in Cinema and the invention of Modern Life / sous la direction de Leo Charney et Vanessa Schwartz. Berkeley, Los Angeles et Londres: University of California Press.

Schwartz (Vanessa). 2017. « Le musée Grévin », p. 50-52 in Dioramas (catalogue d'exposition, Palais de Tokyo, 14 juin - 10 septembre 2017) / sous la direction de Katarina Dohm, Claire Garnier, Laurent Le Bon et Florence Ostende. Paris : Palais de Tokyo et Flammarion.

Séréna-Allier (Dominique). 2016. " Les dioramas du Museon Arlaten : de la lecture critique au projet de conservation/restauration "in situ" ». In Situ. Revue des patrimoines, 29, s. p.

DOI : $10.4000 /$ insitu.13063

Snoep (Nanette), Blanchard (Pascal) \& Boetsch (Gilles) (sous la direction de). 2011. Exhibitions L’invention du sauvage. Paris et Arles : musée du quai Branly - Jacques Chirac et Actes Sud.

Tabart (Marielle) (sous la direction de). 1997. L'Atelier Brancusi. Paris : Centre Pompidou (La collection).

Tamarozzi (Federica). 2017. « Les inventaires du quotidien, pratiques et logiques de collectes ». Tracés, $17, \mathrm{p} .71-84$.

DOI : $10.4000 /$ traces.7120

Thomine-Berrada (Alice). 2015. « Le panorama au XIXe siècle : retour sur un mythe fondateur de la modernité », p. 30-40 in J'aime les panoramas. S'approprier le monde / sous la direction de Laurence Madeline et Jean-Roch Bouiller. Paris : Flammarion.

Vergnet-Ruiz (Jean). 1946. "Château de Compiègne : essai d'une doctrine de restitution des appartements historiques ». Bulletin des musées de France, 11e année, 9, novembre, p. 15-23.

\section{Sources et documents}

Anonyme. 1932. « De M. de Monzie à M. Matisse, tout Paris a défilé au Trocadéro ». Comœdia, 19 juin, p. 3 .

Anonyme. 1934. [Dossier photographique]. Bulletin des musées de France, 1, janvier, p. 24-30.

Alexandre (Arsène). 1931. « La peinture et la sculpture ». La Renaissance, 7, juill., p. 231.

Lami (Eugène Oscar). 1886. Dictionnaire encyclopédique et biographique de l'industrie et des arts industriels (t. II). Paris : Librairie des dictionnaires.

Lemoine (Jean-Gabriel). 1936. «Musées pour le peuple ». L'Écho de Paris, 28 oct., p. 5.

Rivière (George-Henri). 1936. « Les musées de folklore à l'étranger et le futur "musée français des Arts et Traditions populaires » (conférence prononcée le 28 mars 1936 au cours de muséologie de l’École du Louvre). Revue du folklore français et de folklore colonial, mai-juin, p. 58-71.

Rivière (Georges-Henri). 1946. « Musée des Arts et Traditions populaires - Collections d'objets et de documents d'ethnographie française ». Bulletin des musées de France, $11^{\mathrm{e}}$ année, ${ }^{\circ}$ 5, juillet, 

p. 26-44.

Rivière (Georges-Henri). 1973. " Le musée : instrument pour la prise de conscience des problèmes de l'environnement - rôle du musée d'art et du musée de sciences humaines et sociales ». Muséum international, 25(1-2), p. 26-44.

DOI : 10.1111/j.1755-5825.1973.tbo2089.x

Sain (Jules). 1903. Musée rétrospectif du groupe VII - Agriculture - à l'Exposition universelle internationale de 1900 à Paris (rapport). Saint-Cloud : Belin frères.

\section{Notes}

1 Les dix dioramas du MET présentent des mannequins de Pontgibaud (Puy-de-Dôme), d'Attigny (Ardennes), d'Auvergne, de Camargue, de Bourgogne, de Bretagne, des Landes, de Nice, de Provence et de Savoie. Les six unités écologiques du MNATP présentent : un intérieur de basse Bretagne ; la salle commune de la maison des Deuffic, à Goulien (Finistère) ; la forge d'Abraham Isnel, de Saint-Véran (Queyras, Hautes-Alpes) ; l'atelier d'un tourneur, Désiré Louvel (Maine) ; un buron d'Aubrac ; le cabinet du voyant Belline (Paris). Un septième dispositif présente un bateau de pêche de Berck mais qui, par sa nature même, n’a pas été déplacé tel quel de son milieu naturel. Cette évocation, qui joue sur la reconstitution, s'apparente plus à un diorama qu'à une unité écologique.

2 Il est à noter que Georges-Henri Rivière a participé à la conception des salles de synthèse historique, qui précèdent la visite des appartements, sur l'histoire du château de Compiègne. Il a contribué à la mise en place des méthodes historiques et muséographiques employées pour la restitution des appartements.

3 « Du moins, à présent, sommes-nous assurés de posséder dans peu d'années, au voisinage immédiat du Jardin d'acclimatation, un édifice à la mesure de notre programme national. Notre politique d'acquisition s'en trouvera encouragée, voire développée. Car il nous est désormais possible, sans risquer de les enfouir dans un sous-sol inaccessible faute de place pour les exposer, de recréer ces ensembles concrets - intérieurs de demeures paysannes, ateliers d'artisans préindustriels - qui nous faisaient défaut jusqu'ici et dont la présence donnera toute leur valeur éducative, et avec elle plus d'attraits, à nos futures galeries » (Georges-Henri Rivière, 1955, notes tapuscrites, " Avant-propos », in Catalogue nouvelles acquisitions. Exposition "Chefs-d'œuvre de l'art populaire en France » [AN 690AP159]).

4 Georges-Henri Rivière, 7 nov. 1961, « Politique d'accroissement des collections du musée des Arts et Traditions populaires » (AN 690AP).

5 Entreprise Hébert, " mouleur éditeur » (fabricant de mannequins) ; entreprise Wagner (fournisseur des yeux) ; entreprise Dieudonné, perruquier de théâtre (fournisseur des perruques des mannequins) (Archives nationales, dossier comptabilité, AN F/17/3847/1).

6 Ernest Hamy, s. d., « Rapport sur le Musée ethnographique présenté à Monsieur le ministre de l'Instruction publique au nom de la commission spéciale » (AN F/17/3846).

7 « On a pensé qu'il y avait lieu de présenter aux regards des visiteurs quelques tableaux ou scènes », dossier d'œuvre 1907.3 (Mucem, Marseille).

8 Georges-Henri Rivière, 1970, notes tapuscrites, "Muséologie générale contemporaine Fonctions du musée : éducation et exposition » (AN 690AP).

9 Voir le séminaire sur le Magasin de Ben organisé par le musée national d'Art moderne et le Labex CAP, sous la direction de Yaël Kreplack le 12 sept. 2017.

10 Borges (Jorge Luis). 1967. L’Aleph [El Aleph, 1949] (trad. de Roger Caillois et René L.F. Durand). Paris : Gallimard.

\section{Table des illustrations}

\begin{tabular}{|c|c|c|}
\hline & Titre & $\begin{array}{l}\text { Figure 1. Section française du Musée d'Ethnographie du Trocadéro, } \\
\text { Scène d'intérieur : mannequins en costume de Bretagne, Paris, 1884- } \\
\text { 1928, photographie anonyme, ph.1962.205.3 }\end{array}$ \\
\hline & \multirow{2}{*}{$\begin{array}{l}\text { Crédits } \\
\text { URي }\end{array}$} & (C) Mucem \\
\hline & & $\begin{array}{l}\text { http://journals.openedition.org/culturemusees/docannexe/image/2473/img- } \\
\text { 1.jpg }\end{array}$ \\
\hline & \multirow{3}{*}{$\begin{array}{c}\text { Fichier } \\
\text { Titre } \\
\text { Crédits }\end{array}$} & image/jpeg, 312k \\
\hline & & $\begin{array}{l}\text { Figure 2. Tête de mannequin de la section française du Musée } \\
\text { d'Ethnographie du Trocadéro, Paris, vers 1884, Mucem, 2018.41.2 }\end{array}$ \\
\hline & & (C) Mucem \\
\hline & Uي & $\begin{array}{l}\text { http://journals.openedition.org/culturemusees/docannexe/image/2473/img- } \\
\text { 2.jpg }\end{array}$ \\
\hline
\end{tabular}


Fichier image/jpeg, 424k

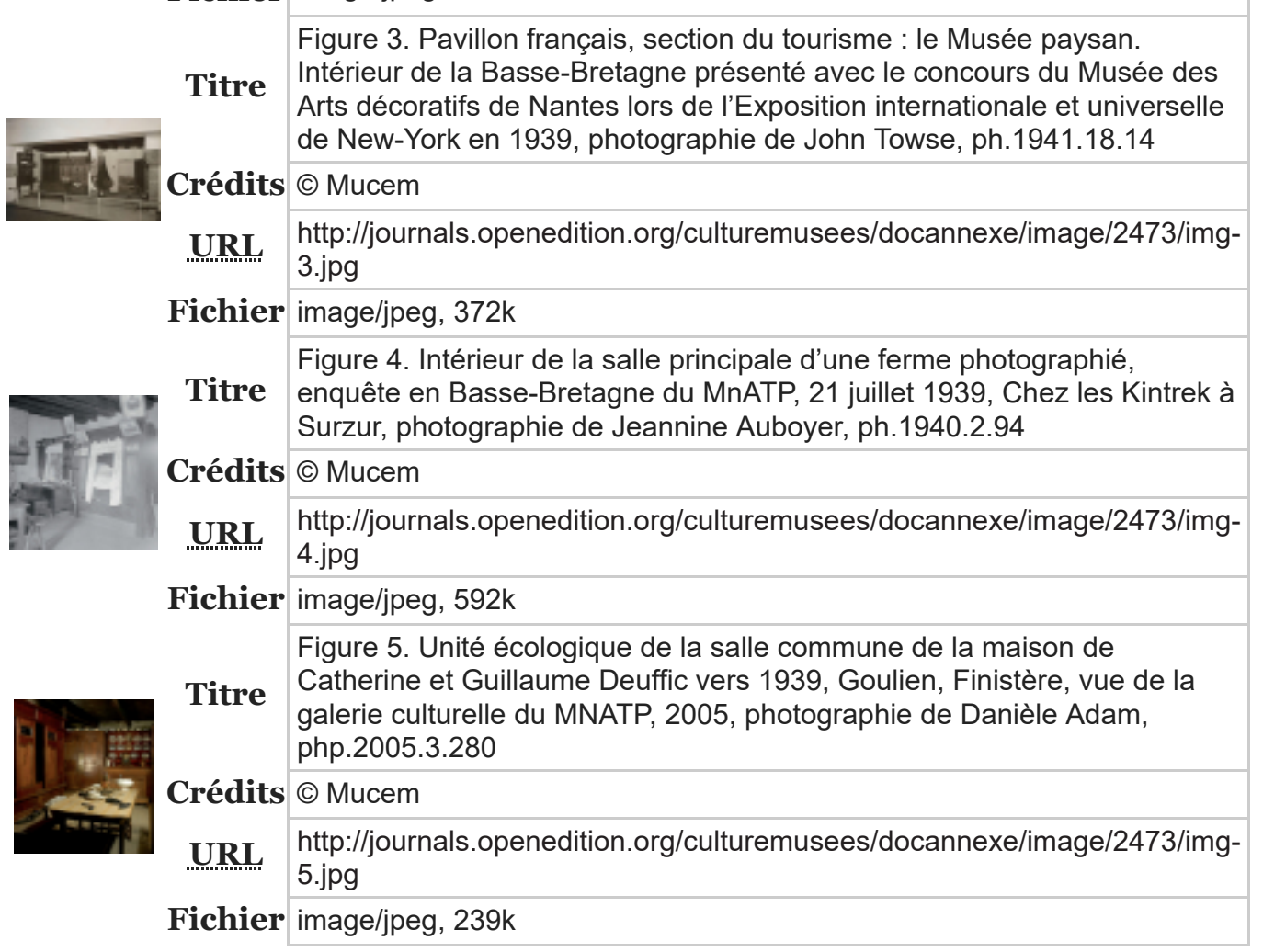

\section{Pour citer cet article}

Référence papier Jean-Roch Bouiller et Marie-Charlotte Calafat, « Dioramas ethnographiques et unités écologiques: La mise en scène de la vie quotidienne au musée d'Ethnographie du Trocadéro et au musée national des Arts et Traditions populaires », Culture \& Musées, 32 | 2018, 131-158.

Référence électronique

Jean-Roch Bouiller et Marie-Charlotte Calafat, « Dioramas ethnographiques et unités écologiques : La mise en scène de la vie quotidienne au musée d'Ethnographie du Trocadéro et au musée national des Arts et Traditions populaires », Culture \& Musées [En ligne], 32 | 2018, mis en ligne le 16 janvier 2019, consulté le 08 juin 2021. URL : http://journals.openedition.org/culturemusees/2473; DOI : https://doi.org/10.4000/culturemusees.2473

\section{Auteurs}

\section{Jean-Roch Bouiller}

Mucem

Jean-Roch Bouiller est docteur en histoire de l'art contemporain et conservateur en chef, responsable du secteur art contemporain au Mucem, depuis 2011. Il a été commissaire de plusieurs expositions à la Galerie d'art du Conseil général des Bouches-du-Rhône à Aix-enProvence et à Sèvres - Cité de la céramique. Au Mucem, il a été commissaire ou commissaire associé des expositions "Au bazar du genre », "La galerie de la Méditerranée », " Des artistes dans la Cité », " Food », "Stefanos Tsivopoulos - History zero », " J'aime les panoramas », "Albanie, 1207 km est », "Graff en Méditerranée », " Document bilingue », " Or ». En plus des catalogues de ces expositions, il a publié de nombreux articles sur l'art contemporain, sur les écrits d'André Lhote, sujet de sa thèse de doctorat, et, avec Françoise Levaillant et Dario Gamboni un livre sur Les bibliothèques d'artistes, XXe-XXle siècles.

Courriel : jean-roch.bouiller[at]mucem.org

\section{Marie-Charlotte Calafat}

Mucem

Marie-Charlotte Calafat est adjointe du département des collections et des ressources documentaires du Mucem, responsable du pôle documentaire et du secteur Histoire du Musée. Au Mucem, elle a été commissaire ou commissaire associée des expositions " Document bilingue », « Roman-Photo » et « Georges Henri Rivière. Voir, c'est comprendre ». Elle est membre du comité de rédaction de la revue Techniques \& Culture.

Courriel : marie-charlotte.calafat[at]mucem.org 

Articles du même auteur

Donner un avenir aux collections du Mucem dans une approche historique [Texte intégral]

Finding a Future for the Mucem's Collections in a Historical Approach

Dar un futuro a las colecciones del Mucem desde un enfoque histórico

Paru dans Culture \& Musées, 37 | 2021

\section{Droits d'auteur}

Culture \& Musées 